\title{
Correlação e causalidade entre os preços de commodities e energia
}

\section{Palavras-chave}

transmissão de preços, cointegração, petróleo, etanol

\section{Classificação JEL}

Q, Q1, Q4

\section{Keywords}

price transmission, cointegration, oil, ethanol

JEL Classification

Q, Q1, Q4
*Doutoranda em Economia Aplicada - ESALQ/USP

**Professor do Departamento

de Ciências Sociais Agrárias da Faculdade de Agronomia Eliseu Maciel - UFPel.

***Professor do Curso de Economia da Universidade Federal de Santa Maria.

\section{Resumo}

Objetiva-se testar a cointegração e a causalidade entre os preços de energia e commodities agrícolas nas condições comercias do Brasil. Utilizando-se dados de preço mensais de 2000 a 2012 das commodities: petróleo, etanol, cana, milho, soja, taxa de câmbio, e ainda os preços americanos de milho e etanol. O petróleo e taxa de câmbio apresentaram coeficientes significativos a $10 \%$ para todos os produtos. $\mathrm{O}$ etanol foi significativo para a cana de açúcar, soja e milho, mostrando o entrelaçamento entre os preços de energia e produtos agrícolas no Brasil. Isso é evidenciado pelas relações significativas de cointegração da soja com a cana, etanol e milho. Identificou-se causalidade no sentido de Granger do milho e etanol americano para o etanol brasileiro, e do milho americano para o milho e a soja do Brasil. Pode-se concluir que há transmissão de preços das commodities energéticas para as commodities agrícolas e entre as commodities.
Dienice Ana Bini*

Mário Duarte Canever ${ }^{* *}$

Anderson Antônio Denardim ${ }^{* * *}$

\section{Abstract}

This paper aims to test and analyze the integration and causality of energy and agricultural commodity prices in the Brazilian context. In order to do that, econometric tests of time series analysis using monthly data, from 2000 to 2012, of the prices of crude oil, ethanol, sugar cane, corn, soybeans and the exchange rate were used. The results show that the prices of petroleum and the exchange rate are the elements most integrated into agricultural prices. The ethanol price is integrated into sugar cane, soybeans and corn prices. Furthermore, soybean prices are also integrated into sugar cane, ethanol and corn, showing that there is a complex and coordinated price mechanism in these markets in Brazil. On the other hand, the Brazilian prices (ethanol, corn and soybean) are caused, in the sense of Granger, by their American counterparts making the Brazilian commodity price formation even more complex. 


\section{1_Introdução}

Os preços das commodities agrícolas são altamente influenciados pelo mercado e afetam de maneira decisiva a renda e a balança comercial do agronegócio, o qual tem expressiva participação no PIB brasileiro. Em 2011, por exemplo, o agronegócio foi responsável por 22,15\% do PIB nacional (Cepea, 2013).

Os preços das principais commodities do agronegócio demonstram instabilidade, com registros de declínio nos anos 2007 e 2008, elevação em 2009, 2010 e início de 2011, alcançando picos históricos em meados de 2012. Apesar da instabilidade, observam-se, nos últimos anos, aumentos reais dos preços da maioria das commodities agrícolas. As recentes elevações registradas podem ser atribuídas a relações de oferta e demanda e ao avanço nos custos de produção (FAO, 2010). Do lado da demanda, notam-se altas motivadas pelo crescimento da população mundial, aumento do poder de consumo nos países em desenvolvimento, principalmente na China e na Índia, e aumento da demanda por atividades agrícolas destinadas à produção de bioenergia (FAO, 2012, 2011, 2010; Brandão, 2012). Do lado da oferta, há redução das quantidades produzidas ocasionadas por fatores climáticos (FAO, 2012), além da baiXa nos estoques mundiais (FAO, 2012; MAPA, 2011). Os preços aumentam também forçados pelo aumento nos custos de produção, advindos da elevação nos preços dos insumos utilizados ao longo das cadeias agroindustriais, a exemplo do petróleo e outras fontes de energia (Baffes, 2007; Chand, 2008; Saghaian, 2010; Margarido et al., 2011).

A elevação nos preços do petróleo aumenta acresce os custos de produção por ser um importante insumo no processo produtivo. Os efeitos vão desde o aumento do custo do transporte a majoração nos preços de insumos agrícolas que utilizam o petróleo e seus derivados na sua constituição.

Outro efeito dos preços do petróleo nos preços agrícolas se dá via ocupação das áreas agrícolas com culturas apropriadas à produção de fontes alternativas de energia, a exemplo da cana de açúcar, no Brasil, e do milho, nos Estados Unidos. A expansão da área cultivada com produtos destinados à obtenção de bioenergia afeta os preços de outras commodities agrícolas por modificar a quantidade ofertada. Tyner (2009) observou, no mercado americano, que, a partir de 2006, o preço do etanol estabeleceu uma ligação entre o preço do petróleo e o do milho, que não existia historicamente.

Além disso, no que tange aos preços, o processo de globalização presenciado pelo mundo, no final do século XX, impulsionou um processo de integração das economias. As interações entre os mercados de commodities podem implicar a presença de volatilidade spillover, que é a transmissão de impulsos comerciais entre os mercados (Cunha, 2008; Santos et al., 2010; Serra et al., 2009; Bellinghini et al., 2011; Caldarelli; Bacchi, 2012). O Brasil tem grande inserção no mercado internacional de produtos agrícolas, visto ser importante exportador de commodities. Assim, com a economia do país mais exposta às agitações internacionais, os preços domésticos podem sofrer mais intensamente as influências dos preços externos (Santos et al., 2007).

$O$ processo de integração de preços entre os diferentes mercados vem acompanhado de outro fenômeno, a integração entre as próprias commodities agrícolas. Por se caracterizarem como bens substitutos no processo produtivo, competindo pelas áreas de cultivos, e muitas vezes serem complementares na fase de consumo, a correlação dos preços e a elasticidade entre as commodities agrícolas são cada vez maiores. A integração entre o preço das commodities agrícolas é evidenciado em vários estudos empíricos, dentre os quais, podem ser citados Libera (2009), Caldarelli e Bacchi (2012), Block et. al. (2012), Zhang et al. (2009) e Saghaian (2010).

Diante da ampla inserção do Brasil no mercado internacional de produtos agrícolas, onde se destaca como expressivo produtor e fornecedor de commodities, os estudos 
relacionados à compreensão das relações entre o setor de energia e os produtos agrícolas tornam-se cada vez mais importantes. Embora muito já tenha sido feito em âmbito nacional, como os trabalhos de Santos et al. (2007,2010), Serra et al. (2009), Brandão (2012) e Bellinghini et al. (2011), nenhum estudo foi dedicado a analisar as relações de cointegração e causalidade entre fontes de energia e commodities agrícolas.

Portanto, objetiva-se neste artigo caracterizar a relação entre os preços de commodities agrícolas (soja, milho e cana-de-açúcar) e os preços de commodities energéticas (petróleo e etanol), nas condições comerciais brasileiras. Examina-se de que forma choques no preço do petróleo e etanol influenciam os preços das commodities agrícolas. Das reflexões anteriores e dos argumentos apresentados na seção de revisão de literatura, derivam-se duas hipóteses principais: (1) movimentos dos preços das commodities energéticas precedem e geram efeitos significativos nos preços das commodities agrícolas;
(2) os mercados das commodities agrícolas estão integrados no âmbito interno ao longo da cadeia agroindustrial, mas também sofrem influências das condições internacionais.

\section{2_Revisão de literatura}

Esta seção dedica-se a apresentar o cenário do setor de energia e o agronegócio, abordando as motivações para a alta nos preços dos alimentos, a crescente integração dos mercados globais e entre as commodities agrícolas.

\section{A alta no preço dos alimentos}

A maior volatilidade e a elevação nos preços dos alimentos é fenômeno observado nos últimos anos. As recentes mudanças de preços têm médias mais elevadas e variações mais amplas que as anteriores (Irwin \& Good, 2009). Isso é comprovado pelo índice de preço de alimentos (Gráfico 1)

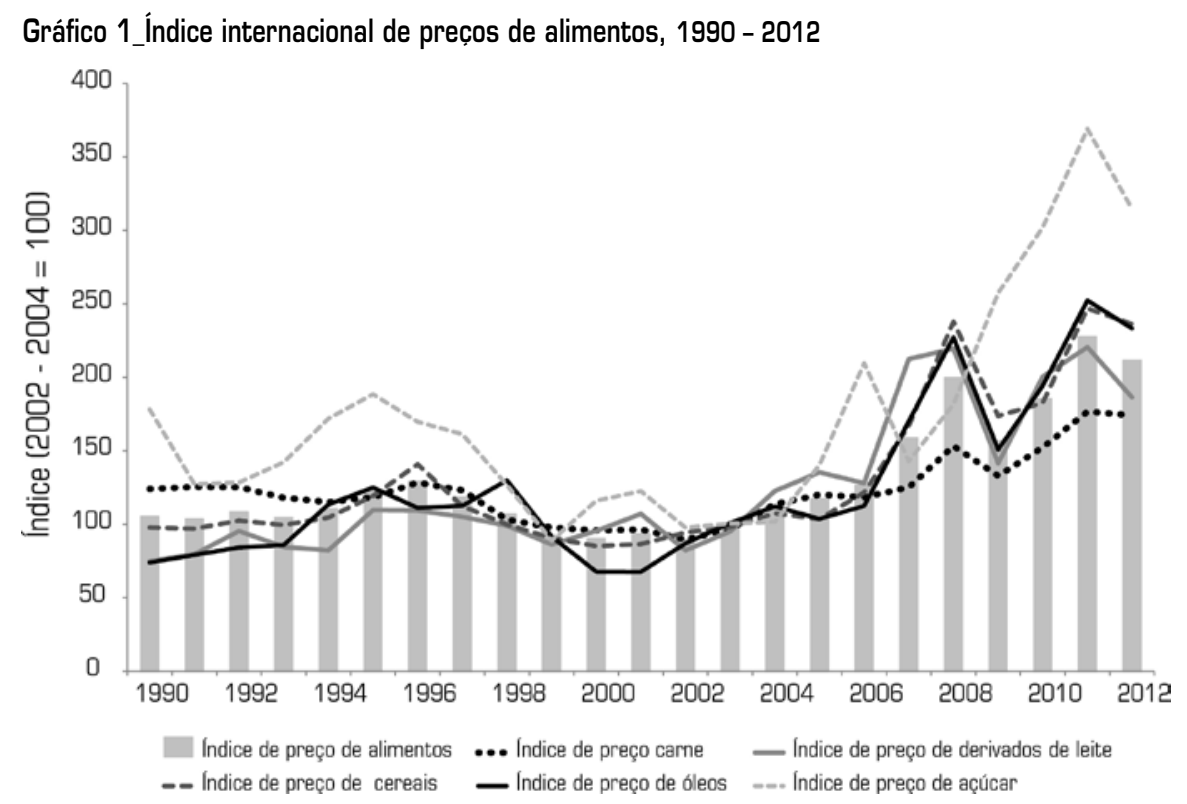

Fonte: Adaptado da FAO (2012). 
estimado pela Organização das Nações Unidas para Agricultura e Alimentação (FAO), com base na média ponderada dos seguintes grupos: carnes (frango, bovina, suína e ovina); derivados de leite (manteiga, queijo e caseína); cereais (trigo, milho e arroz); óleos (média ponderada de 12 diferentes óleos vegetais e de peixe) e açúcar.

Enquanto o período de 1990 a 2003 apresenta variações suaves entre anos sucessivos e sem oscilações expressivas, a partir de 2004 há registro de aumentos mais intensos e maior volatilidade. Isso pode ser constatado pela média e pelo desvio padrão de cada período. De 1990 a 2003, a média do índice foi 105, e o desvio padrão, 12,19, enquanto que, de 2004 a 2012, a média passa para 166, e o desvio padrão, para 42,29.

Como apresentado na seção introdutória, dentre os fatores que contribuem para a elevação dos preços das commodities agrícolas e, consequentemente, a elevação dos preços dos alimentos, podem ser citados o crecimento da demanda mundial impulsionado pelo aumento da população e o aumento da renda per capita em países em desenvolvimento, a integração dos mercados globais, os choques climáticos, a queda nos estoques mundiais, a diminuição do crescimento da produção agrícola, a especulação financeira, os aumentos do preços do petróleo e a expansão dos biocombustíveis (FAO, 2012, 2011, 2010; Brandão, 2012).

Nesta revisão de literatura, dar-se-á maior atenção à relação do preço do petróleo com as commodities agrícolas e às consequências doaumento da produção debiocombustiveis.

Dentre os trabalhos que estudaram as relações entre os preços do petróleo e as commodities agrícolas, Chand (2008) analisou a crise global dos alimentos e afirma que cerca da metade dos aumentos nos preços globais dos alimentos é atribuída a elevação nos preços do petróleo, ocorridos a partir de 2004. Conforme esse autor, o fator primordial na crise de alimentos é a majoração dos preços resultantes tanto da substituição de áreas cultivadas com produtos não alimentícios (bioenergia) quanto do aumento dos custos de produção via custo dos insumos.

Os preços dos fertilizantes, a agricultura mecanizada e os custos de transporte são todos dependentes dos preços do petróleo. Assim, choques nesses produtos são transmitidos para os preços das commodities agrícolas. Pelo fato de o preço do petróleo ter demonstrado volatilidade nos últimos anos, há claramente o potencial de essa volatilidade se espalhar para os preços das commodities (FAO, 2012); Von Braun et al. (2008).

Nesse sentido, Mitchell (2008) encontrou que o preço do petróleo causou um aumento de $15 \%$ a 20\% nos custos de produção e transporte de alimentos nos Estados Unidos. Baffes (2007) assevera que mudanças de preços de commodities energéticas e petróleo têm sido altamente repassadas para commodities alimentares e fertilizantes. Estudo realizado por Saghaian (2010) nas condições norte-americanas observou que há alta correlação entre as séries de preços do petróleo e as commodities agrícolas, como o milho, a soja e o trigo. A presença de transmissão entre o petróleo e o milho, importante commoditie utilizada na alimentação humana, animal e produção de etanol, foi verificada nos trabalhos de Taheripour \& Tyner (2008); Du et al. (2009); Harri e Darren (2009). Nessa linha, Margarido et al. (2011) argumentam que o petróleo e outras commodities tendem a experimentar elasticidades cruzadas cada vez maiores.

O petróleo apresenta também um efeito indireto sobre os produtos agrícolas, já que a elevação do seu preço pode tornar viável a produção de biocombustíveis. Oliveira (2008) aponta que a produção de etanol pelos EUA provocou o aumento do milho no mercado internacional, além de causar o incremento nos preços do arroz, da soja e do trigo, visto que muitos produtores direcionaram a produção agrícola para o milho. Rosegrant (2008) mostrou que 30\% da valoração dos preços dos grãos é resultante da maior demanda por biocom- 
bustíveis. Para o caso do milho, as estimativas desse autor alcançam em torno de $39 \%$ em termos reais, o que demonstra que a maior demanda por biocombustíveis eleva o preço dos produtos agrícolas.

É notável a existência de relações de causalidade entre os preços do petróleo, as fontes alternativas de energia e as demais commodities agrícolas. Como o mercado desses produtos é altamente dinâmico em nível mundial, decorre aí um processo de integração de preços, assunto que será abordado a seguir.

\section{A integração espacial dos mercados agrícolas}

A globalização e o desenvolvimento tecnológico permitiram e facilitaram o maior fluxo de informação e interação entre os mercados. No Brasil, após a abertura comercial na década de 1990 e implementação do Plano Real em 1994, intensifica- ram-se as relações comerciais internacionais, e a agricultura passou a ter papel de destaque na estabilização da economia brasileira.

As exportações do agronegócio brasileiro expandiram significativamente nos últimos anos (Gráf. 2). Com uma parcela expressiva da produção agrícola nacional destinada ao mercado internacional, decorre um processo de importação das condições comerciais externas. A definição dos preços não depende apenas do mercado doméstico, mas da interação das condições nacionais e internacionais.

A intensificação das relações internacionais entre os países deriva de um conceito importante de integração de mercados. Segundo Fackler e Goodwin (2001) e González-Rivera e Helfand (2001), localidades espacialmente separadas que comercializam um mesmo produto formarão um mercado integrado se compartilharem informações e apresentarem

Gráfico 2_Valor e volume das exportações do agronegócio brasileiro de 1997 a 2012

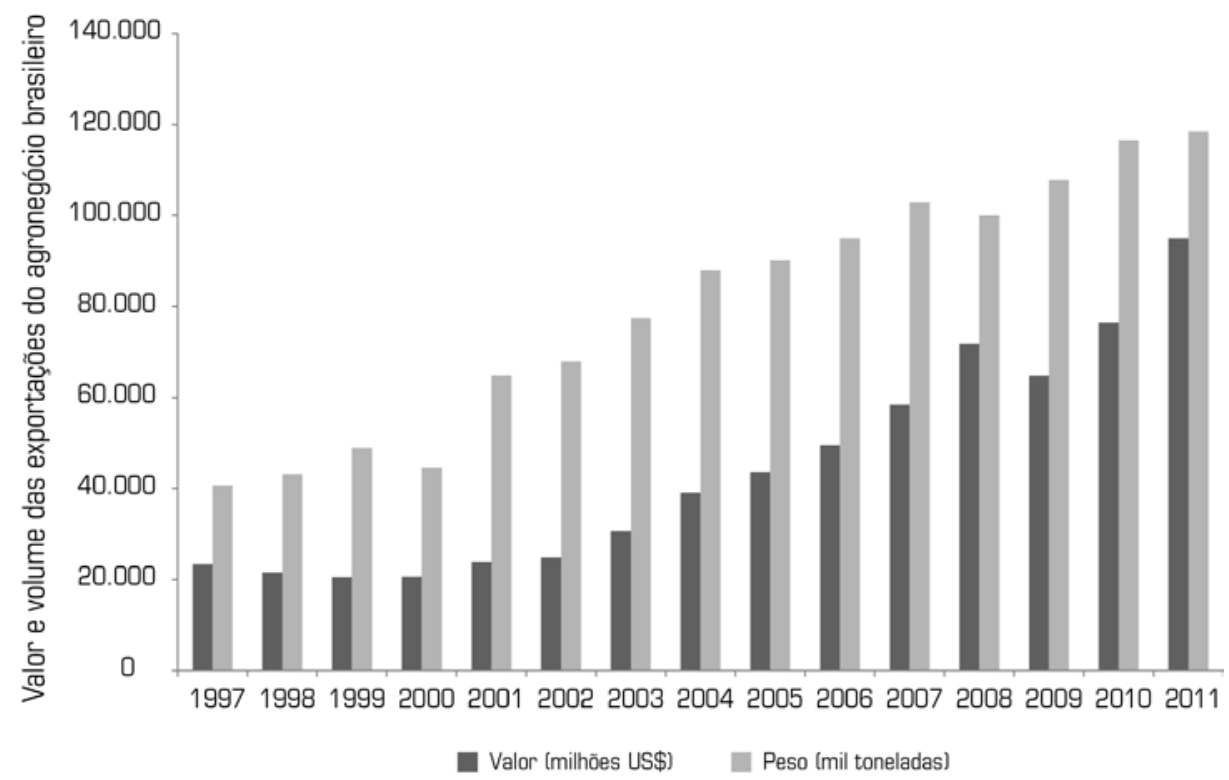

Fonte: Gerado pela autora com base no Agrostat (2012). 
interdependência entre os preços ao longo do tempo. A integração, então, pode ser vista como uma medida do grau em que choques surgidos numa região são transmitidos para outra. Em relação aos mercados agrícolas, Christofoletti et al. (2012) testaram a integração entre os mercados futuros de soja em grão no Brasil, nos Estados Unidos e na China e concluíram que as séries de preços das cotações nesses países são cointegradas, o que comprova a integração entre esses mercados.

Pino e Rocha (1994) concluíram que os preços domésticos no Brasil da soja são fortemente influenciados pelas variações na CBOT. ${ }^{1}$ Margarido et al. (1999) mostraram que variações nos preços da soja em Rotterdam são transferidas para os preços domésticos no Brasil e na Argentina. A partir dessas incursões, fica claro que as cotações brasileiras da soja são altamente influenciadas pelas cotações internacionais, que servem como sinalizadores para o mercado de soja no Brasil.

$\mathrm{Na}$ Tabela 1 é apresentada uma lista de trabalhos que estudaram as relações de integração entre mercados para diferentes produtos agrícolas. Em suma, o que esses trabalhos mostram é que, para vários produtos agrícolas, a hipótese de integração não é descartada. Além disso, outros trabalhos como os de Bellinghini et al. (2011), Santos et al. (2010) e Serra et al. (2009) demonstram que a integração não ocorre apenas entre mercados, mas também entre distintos produtos agrícolas.

Tabela 1_Trabalhos relacionados

\begin{tabular}{|c|c|c|c|}
\hline Autor & Período & Produto & Conclusões \\
\hline $\begin{array}{l}\text { Caldarelli e Bacchi } \\
\text { (2012) }\end{array}$ & 1967 a 2008 & Milho & Existência de interação entre o mercado interno e o externo. \\
\hline Coronel et al. (2010) & 1994 a 2009 & Trigo & $\begin{array}{l}\text { Variações no preço internacional foram, ao longo do tempo, } \\
\text { repassadas quase que completamente para o mercado argentino. }\end{array}$ \\
\hline Sousa e Campus (2009) & & Algodão & Os mercados brasileiro e americano podem ser considerados integrados. \\
\hline Cunha (2008) & 1990 a 2007 & Café & $\begin{array}{l}\text { Integração entre os principais produtores de café arábica e } \\
\text { também entre produtores de café robusta. }\end{array}$ \\
\hline Santos et al. (2007) & 1996 a 2006 & Milho & Causalidade entre o preço internacional do milho e o recebido pelo produtor brasileiro. \\
\hline Silva et al. (2003) & 1995 a 2002 & Milho & $\begin{array}{l}\text { Relações de cointegração entre os preços da soja nos } \\
\text { mercados brasileiro e norte-americano. }\end{array}$ \\
\hline Serra et al. (2009) & 2000 a 2008 & $\begin{array}{l}\text { Petróleo etanol } \\
\text { e açúcar }\end{array}$ & $\begin{array}{l}\text { Aumento nos níveis de preços do petróleo eleva o preço do etanol, } \\
\text { que, por sua vez, faz com que os níveis de preços de açúcar cresçam. }\end{array}$ \\
\hline Bellinghini et al. (2011) & 2010 a 2011 & $\begin{array}{l}\text { Etanol, milho e } \\
\text { petróleo }\end{array}$ & Choques ocorridos no milho e no etanol não foram advindos de choques do petróleo. \\
\hline Santos et al. (2010) & 1994 a 2009 & $\begin{array}{l}\text { Petróleo, etanol } \\
\text { e milho }\end{array}$ & $\begin{array}{l}\text { O preço do milho no Brasil e o do etanol de milho afetam } \\
\text { positivamente o preço do milho no mercado internacional. } \\
\text { O preço do petróleo afeta o preço do milho de forma negativa. }\end{array}$ \\
\hline
\end{tabular}

Fonte: Elaborado pelos autores 


\section{A cointegração entre commodities agrícolas}

Produtos agropecuários apresentam natureza de substituição e/ou complementaridade durante as fases de produção e consumo. Em virtude disso, podem surgir relações de causalidade entre seus preços. Portanto, a formação de preços é condicionada não só pelas relações espaciais dos mercados, mas também por relações causais entre produtos (Tybusch, 2003). Quando variações nos preços de um produto geram respostas em outros, diz-se que há elasticidade cruzada de transmissão de preços.

As evidências de integração entre os preços de commodities agrícolas são demonstradas em trabalhos empíricos, como o estudo realizado por Saghaian (2010). Esse autor concluiu para o mercado norte-americano que há relação de causalidade de Granger uni e bidirecional entre os preços do etanol, do milho, da soja e do trigo.

Embora os estudos que evidenciam a integração entre as commodities agrícolas no Brasil sejam mais escassos do que em esfera internacional, alguns estudos já foram realizados. Tais estudos relacionam os preços de diferentes produtos agrícolas e concluem, por exemplo, que há transmissão de preços entre os mercados físico da soja e futuro do milho (Libera, 2009), assim como interação no mercado físico desses dois produtos (Caldarelli; Bacchi, 2012). Na mesma perspectiva, Block et al. (2012) concluíram que o etanol hidratado influencia o preço da cana e do açúcar no Estado de São Paulo.

Ao longo desta revisão de literatura, foi possível demonstrar,com base em estudos empíricos, que o aumento no preço do petróleo é transmitido para o preço dos produtos agrícolas. A causa desse efeito se dá pela relação insumo produto existente nesses mercados. Além disso, o aumento dos preços do petróleo estimula o desenvolvimento de fontes alternativas de energia, dentre elas os biocombustíveis produzidos a partir de massa vegetal, e o consequente aumento da demanda por produtos agrícolas como soja, milho e cana-de-açúcar. Como, no nível da produção agrícola, esses produtos competem por área, a elevação da demanda aumenta o grau de integração entre seus preços. Somado a isso, têm-se a integração dos mercados globais decorrente da ascensão das relações comerciais entre os países.

\section{3_Metodologia}

Para testar as duas hipóteses deste estudo - (1) movimentos dos preços das commodities energéticas precedem e geram efeitos significativos nos preços das commodities agrícolas e; (2) os mercados das commodities agrícolas estão integrados no âmbito, mas também sofrem influências das condições internacionais -, usou-se o ferramental econométrico de séries temporais. Especificamente, utilizam-se os modelos (VAR e VEC) para testar as hipóteses de dependência de preços entre commodities energéticas e agrícolas e entre as próprias commodities agrícolas, e os testes de causalidade de Granger e função impulso-resposta como testes complementares para testar as hipóteses. Também se utiliza o teste de Causalidade de Granger para testar a hipótese que o mercado interno é causado no sentido de Granger pelo mercado externo, assim como a existência de precedência nos movimentos de preços das commodities energéticas em relação às commodities agrícolas.

Como alguns produtos são destinados ao mercado externo ou oriundos do mercado externo, optou-se por incluir a taxa de câmbio como forma de filtrar os efeitos das variações cambiais sobre os preços internos. Os dados de preços do petróleo, etanol americano e milho americano foram convertidos de dólar para real, tendo como base a taxa de câmbio comercial-compra-média coletada no Ipeadata. Foram coletados dados mensais de janeiro de 2000 a julho de 2012. 
Quadro 1_Apresentação das variáveis utilizadas neste estudo

\begin{tabular}{l|l|l} 
Variável & Descrição & Local de coleta \\
\hline Petróleo & Logaritmo natural do preço do petróleo & FMI \\
\hline Cana & $\begin{array}{l}\text { Logaritmo natural do preço da cana-de- } \\
\text { açúcar - preço recebido pelo produtor }\end{array}$ & IEA \\
\hline Etanol & $\begin{array}{l}\text { Logaritmo natural do preço do etanol } \\
\text { brasileiro - preço recebido pelo produtor }\end{array}$ & IEA \\
\hline Milho & $\begin{array}{l}\text { Logaritmo natural do preço do milho } \\
\text { brasileiro - preço recebido pelo produtor }\end{array}$ & IEA \\
\hline Soja & $\begin{array}{l}\text { Logaritmo natural do preço da soja } \\
\text { brasileiro - preço recebido pelo produtor }\end{array}$ & IEA \\
\hline Corn & $\begin{array}{l}\text { Logaritmo natural do preço do milho } \\
\text { americano - preço recebido pelo produtor }\end{array}$ & USDADATE \\
\hline Ethanol & $\begin{array}{l}\text { Logaritmo natural do preço do etanol } \\
\text { americano - preço recebido pelo produtor }\end{array}$ & USDADATE \\
\hline Câmbio & $\begin{array}{l}\text { Logaritmo natural da taxa de câmbio } \\
\text { comercial-compra-média }\end{array}$ & IPEADATA \\
\hline
\end{tabular}

Fonte: Elaborado pelos autores

As variáveis utilizadas neste estudo estão sumarizadas no Quadro 1.

Como se tratou de séries de preços agrícolas, procedeu-se ao exame de sazonalidade por meio do método de regressão simples, com a inserção de 11 variáveis dummies, correspondendo a uma dummy para cada mês. O passo seguinte foi testar a estacionariedade das séries usando para tal os métodos de Augmented Dickey-Fuller (ADF) e Dickey Fuller-Mínimos Quadrados Generalizados (DF-GLS), versão eficiente do teste Dickey-Fuller Aumentado, que consiste na aplicação do teste ADF à série previamente filtrada de seus componentes determinísticos. Posteriormente, testou-se a cointegração das séries usando o modelo de Johansen \& Juselius (1990), o qual se baseia em dois testes, a estatística do traço $(\lambda$ traço) e a estatística da máxima $(\lambda \max )$, que apresentam a forma reduzida, como segue na Equações $1 \mathrm{e}$ 2, respectivamente:

$$
\begin{aligned}
& \lambda_{\text {traco }}=-T \sum_{i=1+r}^{k}\left(\left[\log \left(1-\lambda_{r+1}\right)\right]\right) \\
& \lambda_{\max }=-T \log \left(1-\lambda_{r+1}\right)
\end{aligned}
$$

De posse dos resultados do teste de cointegração, deve construído um Vetor Autorregressivo (VAR), caso não existam vetores cointegrantes, ou Vetores de Correção de Erros (VEC), se existirem vetores cointegrantes. O modelo VAR pode ser apresentado na forma reduzida conforme Equação 3. A equação com a inclusão do vetor de correção é representada pela Equação 4 .

$$
\begin{aligned}
& X_{t}=\alpha+\sum_{j=1}^{m} \beta_{j} X_{t-j}+\sum_{j=1}^{m} \gamma_{j} Y_{t-j}+u_{1 t} \\
& \Delta y_{t}=a_{0}+b_{1} \Delta X_{t} \pi \beta_{t-1}+e_{t}
\end{aligned}
$$

A inclusão de um vetor de correção de erros no Vetor Autorregressivo captura a informação de longo prazo contida na tendência estocástica de cada série. Além disso, a estimação das elasticidades permitirá inferir por meio da significância dos coeficientes de longo prazo, se existe transmissão entre as variáveis testadas, dado que se utilizaram as séries em logaritmo natural. Assim, a presença de coeficientes significativos indicará a existência de transmissão de preços, enquanto o valor do coeficiente indicará a elasticidade, ou seja, se uma variação de $1 \%$ numa variável explicativa é transmitida mais ou menos que proporcionalmente para o preço da variável explicada.

A última etapa consiste na realização do teste de causalidade de Granger ${ }^{2}$ e da função impulso-resposta. Como as séries dos modelos são cointegradas, utilizou-se o teste de causalidade proposto por Engle e Granger, que modifica o padrão de causalidade na medida em que incorpora um possível efeito de longo prazo na análise. $O$ método baseado no mecanismo de correção de erros examina se valores defasados de uma variável $x$ podem ajudar a explicar 
mudanças nos valores correntes de outra variável $y$, mesmo se mudanças passadas em $y$ não sejam relevantes (Carneiro, 2012). Dessa forma, a presença significativa de causalidade de Granger entre as variáveis permitirá identificar relações de precedência de variação de uma variável em relação à outra. A função impulso-resposta permite estimar como uma variável responde a choques não esperados na própria e nas demais variáveis.

O número de defasagens dos modelos foi determinado com base no critério de informação de Akaike (AIC), no critério de Schwarz (SC) e no critério de informação de Hannam-Quinn (HQ). Nos modelos estimados, priorizou-se a inexistência de autocorrelação dos resíduos, a qual foi verificada até $\mathrm{a} 12^{\mathrm{a}}$ defasagem, com auxílio do teste de Ljung-Box. Considerando que o objetivo do trabalho é conhecer o comportamento das séries de preços agrícolas, não serão apresentados os resultados para a taxa de câmbio e petróleo.

\section{4_Resultados e discussão}

\section{Os testes de adequabilidade dos modelos}

Como as séries não são sazonais, partiu-se diretamente para a verificação de existência de raiz unitária. Em uma série não estacionária, a média e a variância apresentam dependência temporal, o que acarreta erros de estimação. Conforme análise realizada com auxílio dos testes Aumentado de Dickey Fuller (ADF) e Dickey Fuller-Mínimos Quadrados Generalizados (DF-GLS) e apresentada na Tabela 2, as séries em nível são não estacionárias. Quando tomadas em primeira diferença, porém, tornam-se estacionárias, ou seja, a hipótese nula de existência de raiz unitária é rejeitada ao nível de $1 \%$ de significância.

As Tabelas 3 e 4 apresentam o resultado dos testes de cointegração de Johansen. $\mathrm{O}$ valor crítico menor que a esta-
Tabela 2_Resultados dos testes ADF e DF-GLS em nivel para as séries mensais das séries logaritmizadas, janeiro de 2000 a junho de 2012

\begin{tabular}{|c|c|c|c|c|c|c|}
\hline \multirow{2}{*}{ Variáveis } & \multicolumn{3}{|l|}{$A D F^{a}$} & \multicolumn{3}{|c|}{ DF-GLS } \\
\hline & Nivel & 1 a diferença & t crítico ${ }^{c}$ & Nivel & $1^{\text {a }}$ diferença & $t$ crítico \\
\hline Petróleo & $-1,93$ & $-12,30$ & $-3,47$ & $-0,09$ & $-11,35$ & $-2,58$ \\
\hline Câmbio & $-1,84$ & $-7,84$ & $-3,47$ & $-1,43$ & $-7,45$ & $-2,58$ \\
\hline Soja & $-1,71$ & $-7,45$ & $-3,47$ & $-0,32$ & $-7,06$ & $-2,58$ \\
\hline Cana & $-2,56$ & $-12,49$ & $-3,47$ & $-0,33$ & $-12,48$ & $-2,58$ \\
\hline Milho & $-2,41$ & $-7,52$ & $-3,47$ & $-2,26$ & $-6,22$ & $-2,58$ \\
\hline Etanol & $-3,01$ & $-1,01$ & $-3,47$ & $-0,87$ & $-9,48$ & $-2,58$ \\
\hline Corn & $\mid-1,47$ & $-8,12$ & $-3,47$ & $-0,05$ & $-8,04$ & $-2,58$ \\
\hline Ethanol & $-3,27$ & $-10,06$ & $-3,47$ & $-0,92$ & $-9,99$ & $-2,58$ \\
\hline
\end{tabular}

Fonte: Resultados da pesquisa.

Nota: a: Teste Dickey-Fuller Aumentado; b: Dickey Fuller-Mínimos Quadrados Generalizados; c: Valores críticos com 1\% de significância;

Tabela 3_Teste do traço para cointegração das séries, janeiro de 2000 a junho de 2012

\begin{tabular}{r|r|r|r|r} 
Hipótese nula & Autovalor & Estatística traço & $\begin{array}{l}\text { Valor crítico } \\
\text { a } 5 \%\end{array}$ & Prob. * * \\
\hline$r=0 *$ & 0,312 & 118,844 & 107,347 & 0,007 \\
\hline$r \leq 1$ & 0,157 & 63,510 & 79,341 & 0,422 \\
\hline$r \leq 2$ & 0,097 & 38,183 & 55,246 & 0,611 \\
\hline$r \leq 3$ & 0,079 & 23,137 & 35,011 & 0,500 \\
\hline$r \leq 4$ & 0,041 & 10,906 & 18,398 & 0,397 \\
\hline$r \leq 5$ & 0,031 & $\ldots .6,685$ & $\ldots, 841$ & 0,030
\end{tabular}

Fonte: Resultados da pesquisa.

Nota: a: Considerou o modelo: Intercepto e tendência na equação de cointegração e intercepto no VAR (modelo 4 do Eviews); ${ }^{\star}$ Denota a rejeição da hipótese nula ao nível de $5 \%$ de significância; ${ }^{* *}$ Valores confrontados com o teste de MacKinnon-Haug-Michelis (1999).

tística calculada para o teste do traço (Tabela 3) aponta para a rejeição da hipótese nula de zero vetor cointegrante. Já a hipótese de no máximo um vetor não pode ser rejeitada, 
Tabela 4_Teste do Máximo Autovalor para cointegração das séries, janeiro de 2000 a junho de 2012

\begin{tabular}{r|r|r|r|r} 
Hipótese nula & Autovalor & Estatística traço & $\begin{array}{l}\text { Valor crítico } \\
\text { a } 5 \%\end{array}$ & Prob. * * \\
\hline$r=0 *$ & 0,312 & 55,334 & 43,420 & 0,002 \\
\hline$r=1$ & 0,157 & 25,327 & 37,164 & 0,566 \\
\hline$r=2$ & 0,097 & 15,046 & 30,815 & 0,899 \\
\hline$r=3$ & 0,079 & 12,231 & 24,252 & 0,745 \\
\hline$r=4$ & 0,041 & 6,221 & 17,148 & 0,795 \\
\hline$r=5$ & 0,031 & $\ldots, 685$ & 3,841 & 0,030
\end{tabular}

Fonte: Resultados da pesquisa.

Nota: a: Considerou o modelo Intercepto e tendência na equação de cointegração e intercepto no VAR (modelo 4 do Eviews); ${ }^{\star}$ Denota a rejeição da hipótese nula ao nível de $5 \%$ de significância; ${ }^{* *}$ Valores confrontados com o teste de MacKinnon-Haug-Michelis (1999).

indicando que existe um vetor de cointegração entre as variáveis do modelo.

De acordo com o resultado do teste do máximo autovalor (Tabela 4), a hipótese nula de que o posto da matriz de cointegração é nulo $(\mathrm{r}=0)$ é rejeitado ao nível de significância de $5 \%$. Desse modo, é possível concluir que há um vetor de cointegração que estabelece as relações de equilíbrio de longo prazo entre as variáveis.

Portanto, de acordo com os dois testes, existe um vetor de cointegração que estabelece relação de equilíbrio de longo prazo entre as variáveis estudadas. Valendo-se dessa constatação, foi estimado o vetor de correção de erro - VEC (1) e normalizado para as variáveis agrícolas de interesse do modelo (Tabela 5).

É importante destacar que a ordenação das variáveis foi realizada valendo-se do teste de exogeneidade de Wald Block Exogenety, conforme Enders (1995), com base no valor da estatística Qui-Quadrado. As variáveis com menor valor da estatística (mais exógenas) são colocadas antes das vari- áveis mais endógenas, de acordo com a ordem que aparece para cada variável, na Tabela 5. O teste de Ljung-Box não identificou a presença de autocorrelação dos resíduos até a $12^{\mathrm{a}}$ defasagem.

\section{Resultados empíricos}

As estimativas dos coeficientes de longo prazo do modelo vetorial de correção de erro (Tabela 5) mostram que variações nos preços do etanol são transferidas menos que proporcionalmente para a cana-de-açúcar. O que pode justificar tal inelasticidade é que a cana é matéria-prima direta para a produção de dois produtos igualmente importantes, o etanol e o açúcar; assim a formação de seu preço deve ser resultado do comportamento dos preços desses dois produtos. Além disso, $82,37 \%$ da moagem é realizada em unidades mistas (produzem tanto açúcar quanto etanol), indicando alta flexibilidade entre a produção de etanol e a de açúcar, conforme as condições do mercado (Conab, 2012).

A mesma justificativa é válida para o coeficiente significativo e elástico da cana na equação de cointegração do etanol. Uma vez que a produção de cana é dividida entre a produção de etanol e a de açúcar, variações no preço da matéria-prima são transmitidas um pouco mais que proporcionalmente ao preço do produto. Esses resultados também podem ser interpretados como uma forma de poder de mercado, uma vez que variações no preço do etanol são transmitidas menos que proporcionalmente para o preço da cana (matéria-prima), ao passo que variações no preço da cana são transmitidas mais que proporcionalmente para o preço do etanol (produto), o que é amplamente aceitável para uma cadeia onde existem muitos vendedores e poucos compradores.

O petróleo e a taxa de câmbio apresentaram coeficientes significativos em todas as equações de cointegração. Esses resultados mostram a importância do petróleo como com- 
Tabela 5_Vetor de cointegração normalizado para cada variável do modelo

\begin{tabular}{|c|c|c|c|c|c|c|c|}
\hline Cana & C & Soja & Milho & Câmbio & Etanol & Petróleo & Tend \\
\hline \multirow[t]{2}{*}{1,000} & $-4,702$ & $0,313 *$ & $-0,082$ & $-0,477^{\text {关关兑 }}$ & 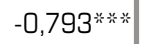 & $0,177 \%$ & $-0,006$ \\
\hline & & $(1,864)$ & $(-0,625)$ & $(-2,955)$ & $(-6,846)$ & $(1,646)$ & $(-3,249)$ \\
\hline Etanol & C & Milho & Câmbio & Petróleo & Soja & Cana & Tend \\
\hline \multirow[t]{2}{*}{1,000} & 5,931 & 0,103 & 0,601 灾前 & $-0,223^{*}$ & $-0,395^{*}$ & $-1,261 * \% *$ & 0,007 \\
\hline & & $(0,636)$ & (2,700) & $(-1,648)$ & $(-1,794)$ & $(-7,156)$ & (2,777) \\
\hline Milho & $C$ & Petróleo & Etanol & Cana & Soja & Câmbio & Tend \\
\hline \multirow[t]{2}{*}{1,000} & 5,761 & $-2,171^{*}$ & 9,714 \%** & $-12,253^{\text {羊前 }}$ & $-3,839 \%$ & 5,840 兼 & 0,070 \\
\hline & & $(-1,865)$ & $(6,443)$ & $(-6,617)$ & $(-2,236)$ & $(2,897)$ & $(2,911)$ \\
\hline Soja & $C$ & Petróleo & Cana & Milho & Câmbio & Etanol & Tend \\
\hline \multirow[t]{2}{*}{1,000} & $-15,005$ & $0,565^{*}$ & 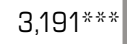 & $-0,260$ & $-1,521 \%$ & $-2,530 \%$ & $-0,018$ \\
\hline & & $(1,700)$ & $(7,075)$ & {$[-0,801)$} & $(-4,246)$ & $(-6,514)$ & $(-4,469)$ \\
\hline
\end{tabular}

Fonte: Resultado da pesquisa.

Nota: ${ }^{* \star}$ Valores significativos a $1 \% ;{ }^{* \star}$ Valores significativos a $5 \% ;{ }^{*}$ Valores significativos a $10 \% . \mathrm{O}$ valor entre parênteses refere-se ao valor t.

modity referência de preço, fato que já é bem conhecido na literatura (Saghaian, 2010; Du et al., 2009; Chand, 2008; Harri; Darren, 2009; Mitchell, 2008; Taheripour \& Tyner, 2008; Von Braun et al., 2008; Baffes, 2007). Já a significância da taxa de câmbio demonstra que, em razão da grande inserção do Brasil no comércio mundial de produtos agrícolas, essa variável é relevante na determinação dos preços internos, até mesmo dos produtos que não são diretamente exportados, como a cana.

O preço da soja é significativo para o preço do milho; isso se justifica pela relação de substituição entre esses produtos na fase de produção. Como já se sabe, a persistente apreciação da soja no mercado internacional estimula a expansão da área cultivada com soja em detrimento do milho, acarretando oferta menor desse produto e contribuindo para o aumento dos seus preços. A relação inversa não se observa.
Outro resultado interessante é a significância estatística do etanol e da cana nas equações de cointegração da soja e do milho. E, de maneira recíproca, da soja nas equações da cana e do etanol. A expectativa sobre tal resultado é que a relação significativa encontrada mostre que variações de preços no mercado americano de milho e/ou do etanol se propagam para o mercado brasileiro de etanol e/ou de cana-de-açúcar. Isso, portanto, reforça a hipótese de mercados cada vez mais integrados entre commodities, conforme já salientado por Santos et al. (2007); Coronel et al. (2010); Block et al. (2012). Essa hipótese será testada mais adiante neste trabalho (Tabela 8). Corrobora com esse argumento o fato de a intensidade de transmissão do etanol e da cana ser mais forte para o milho do que para a soja.

Além das relações de equilíbrio de longo prazo, podem ocorrer desequilíbrios de curto prazo, segundo os coefi- 
cientes do VEC, apresentados na Tabela 6. Em relação aos parâmetros de curto prazo, a cana foi o único produto do qual o próprio valor defasado não colabora para a correção dos desequilíbrios. Tal fato reforça a relevância dos seus derivados para a formação do preço, uma vez que o etanol com aproximadamente $15 \%$ dos desequilíbrios transitórios no preço da cana a cada período. Outro elemento que ajuda significativamente no retorno do mercado da cana para a situação de equilíbrio é o mercado de petróleo. Isso demonstra, portanto, que o equilíbrio do mercado da cana é dependente dos mercados dos produtos energéticos (etanol e petróleo). Em relação ao etanol, o equilíbrio de curto prazo depende unicamente da movimentação dos próprios preços defasados.

\section{Tabela 6_Coeficientes do VEC}

\begin{tabular}{|c|c|c|c|c|}
\hline \multirow{2}{*}{$\begin{array}{l}\text { Variáveis } \\
\text { explicativas }\end{array}$} & \multicolumn{4}{|c|}{ Variáveis explicadas } \\
\hline & D(Cana) & D(Etanol) & D(Milho) & $D($ Soja) \\
\hline \multirow[t]{2}{*}{$D($ Cana $(-1))$} & 0,043 & $-0,148$ & $-0,038$ & $-0,037$ \\
\hline & $(0,467)$ & $(-1,590)$ & $(-0,642)$ & $(-0,776)$ \\
\hline \multirow[t]{2}{*}{ D(Soja(-1)) } & $-0,076$ & $-0,053$ & 0,081 & $0,282 \%$ \\
\hline & {$[-0,432]$} & $(-0,292)$ & $(0,705)$ & $(3,033)$ \\
\hline \multirow[t]{2}{*}{ D(Milho(-1)) } & 0,060 & $-0,021$ & $0,345 *$ & 0,086 \\
\hline & $(0,454)$ & $(-0,153)$ & $(4,036)$ & $(1,235)$ \\
\hline \multirow[t]{2}{*}{ D(Câmbio(-1)) } & 0,139 & $-0,049$ & $0,279 \%$ & 0,193 *\%* \\
\hline & $(0,665)$ & $(-0,230)$ & $(2,057)$ & $(1,751)$ \\
\hline \multirow[t]{2}{*}{$D($ Etanol(-1)] } & $-0,149$ *** & $0,224 \%$ & $-0,008$ & $-0,102 *$ \\
\hline & $(-2,050)$ & $(3,020)$ & {$[-0,179)$} & $(-2,653)$ \\
\hline \multirow[t]{2}{*}{$D($ Petróleo(-1)] } & $0,213^{* * *}$ & 0,024 & $-0,005$ & $-0,004$ \\
\hline & $(2,487)$ & $(0,274)$ & $(-0,094)$ & $(-0,084)$ \\
\hline \multirow[t]{2}{*}{ C } & 0,007 & 0,007 & 0,001 & 0,006 \\
\hline & $(0,886)$ & $(0,797)$ & $(0,227)$ & $(1,443)$ \\
\hline
\end{tabular}

Fonte: Resultados da pesquisa.

Nota: ${ }^{* *}$ Valores significativos a $1 \%$; ${ }^{*}$ Valores significativos a $5 \%$; ${ }^{\star}$ Valores significativos a $10 \%$. $\mathrm{O}$ valor entre parênteses refere-se ao valor $\mathrm{t}$.
Por sua vez, os desequilíbrios de curto prazo do milho e da soja são corrigidos pelos próprios preços defasados e uma contribuição do petróleo, e, no caso da soja, também pelo etanol. Obviamente, como discutido, para o longo prazo, a contribuição do etanol no caso brasileiro não se dá por razões espúrias, e sim por resultado da integração dos mercados brasileiro e norte-americano de milho e etanol. Nesse ponto, é possível verificar o entrelaçamento entre os mercados de alimentos e bioenergia em âmbito mundial. Tal fato já havia sido constatado por outros autores, como Margarido et al., (2011) e Christofoletti et al. (2012). Com demanda crescente em nível mundial e escassez de área agrícola para a produção de alimentos e energia, há uma pressão generalizada nos preços dos alimentos, o que gera vasos comunicantes que interligam os mercados de alimentos e energia. Os coeficientes indicam uma correção em direção ao equilíbrio entre $15 \%$ e $30 \%$ a cada mês.

As relações de causalidade de curto prazo verificadas por meio do teste de causalidade de Granger (Tabela 7) foram pouco significativas. Apenas o petróleo e o etanol causam no sentido de Granger a cana. Não se verificou a presença de causalidade bidirecional. Esperava-se que variações no preço de petróleo precedessem variações significativas nos preços dos demais produtos, já que o petróleo entra como insumo na produção agrícola. A não significância pode estar refletindo as características intrínsecas da agricultura, segundo Nerlove (1958), a produção agrícolas e, consequentemente, os preços apresentam certa impossibilidade de se ajustarem a variações nos preços dos próprios produtos, produtos concorrentes e insumos utilizados na produção agrícola no curto prazo, graças às características sazonais.

Antes de prosseguir com as análises e apresentar a função impulso-resposta entre as séries, foram analisadas as relações entre os preços nacionais de milho, etanol e soja e os preços americanos de milho e etanol. As cinco séries são 
Tabela 7_Teste de causalidade baseado no VEC

\begin{tabular}{|c|c|c|c|c|c|}
\hline \multirow{2}{*}{ Var. dependente } & \multicolumn{5}{|c|}{ Curto independentea (variável explicativa) } \\
\hline & [Corn] & $\mathrm{D}$ (Ethanol) & D(Etanol) & D(Milho) & $D($ Soja) \\
\hline $\mathrm{D}$ (Corn) & --- & 0,2910 & 0,7828 & 0,8971 & 0,1788 \\
\hline D(Ethanol) & $0,0195^{* * *}$ & --- & 0,2497 & 0,3476 & 0,3166 \\
\hline $\mathrm{D}$ (Etanol) & $0,0507 *$ & 0,0008 等 & --- & $0,0667 *$ & 0,2219 \\
\hline D(Milho) & $0,0626 *$ & 0,2479 & 0,7870 & --- & 0,1566 \\
\hline$D$ (Soja) & $0,0617 *$ & 0,1917 & $0,0582 *$ & 0,1609 & --- \\
\hline
\end{tabular}

Fonte: Resultado da pesquisa.

Nota: a: corresponde ao p-valor de teste de Wald Block Exogenety; b: coeficiente do termo de correção de erro; ${ }^{* * *}$ Valores significativos a $1 \%$; ${ }^{* *}$ Valores significativos a $5 \%$; ${ }^{\star}$ Valores significativos a $10 \%$.

integradas de ordem 1 - I(1). O número de defasagem escolhido para o VAR, que contém as cinco séries (milho brasileiro, milho americano, soja brasileira, etanol brasileiro e etanol americano) foi de apenas um lag. 0 teste de Johansen aponta para a existência de um vetor de cointegração (existência de intercepto na equação de cointegração e no VAR).

$\mathrm{O}$ milho americano Granger causa o milho brasileiro. O etanol brasileiro é causado no sentido de Granger pelo milho brasileiro e pelo etanol e milho americano. Os resultados considerando apenas os preços do milho e do etanol poderiam ser representados como uma pirâmide, onde o milho americano estaria na parte superior (como a variável mais exógena), e o etanol brasileiro, na base (como a variável mais endógena). Quanto à soja, observa-se que essa recebe extravasamentos do etanol brasileiro e do milho americano, reforçando a hipótese de integração tanto entre mercado quanto entre commodities. O milho americano (CORN) não é causado no sentido de Granger por nenhuma das demais variáveis. Já o etanol americano (ETHANOL) é causado apenas pelo milho americano, não recebendo influência dos preços brasileiros. É evidente que variações nos preços americanos precedem variações nos preços brasileiros.

A existência de um vetor de cointegração e de causalidade no sentido de Granger dos preços americanos em relação aos preços brasileiros são indicações de que os preços naquele país servem como referência para o mercado interno, o que confirma a hipótese (2) de que os preços domésticos recebem influência dos preços internacionais, especialmente os americanos.

O Gráfico 3 apresenta a função impulso-resposta das variáveis do modelo e deve ser interpretada da seguinte maneira, tomando o primeiro gráfico como exemplo, 'resposta da cana a um choque não antecipado nas demais séries e no próprio preço. Observa-se que os choques promovem, na maioria, reações positivas, o que é esperado por causa das relações insumos/produtos ou de substitutibilidade entre as variáveis. As reações das variáveis após um choque no (seu) próprio preço são persistentes, exceto a do etanol. A intensidade de reação aos choques pode ser considerada moderada, pois não ultrapassa $10 \%$ em todos os casos.

Valendo-se dos resultados obtidos, é possível concluir que há dependência tanto do etanol quanto das commodities agrícolas no mercado brasileiro em relação ao preço internacional do petróleo. Também foi possível confirmar a hipótese de entrelaçamento das commodities agrícolas ao preço do etanol. Já entre os preços das commodities agrícolas não há regra, sendo que a sujeição de preços ocorre ou não de acordo com cada produto. Por fim, foi possível verificar vinculação no curto prazo dos preços brasileiros de milho, soja e etanol e os preços americanos de milho e etanol, através dos valores significativos do teste de causalidade de Granger. A relação inversa não se verifica.

Com base nos resultados obtido nos testes, principalmente no VEC (1), é possível aceitar a hipótese 1 de que variações nos preços das commodities energéticas são repassa- 
Gráfico 3_Função impulso-resposta das variáveis no modelo diante de choques não esperados na própria e nas demais variáveis

Response of Cana to Cholesky_ One S.D. Innovations

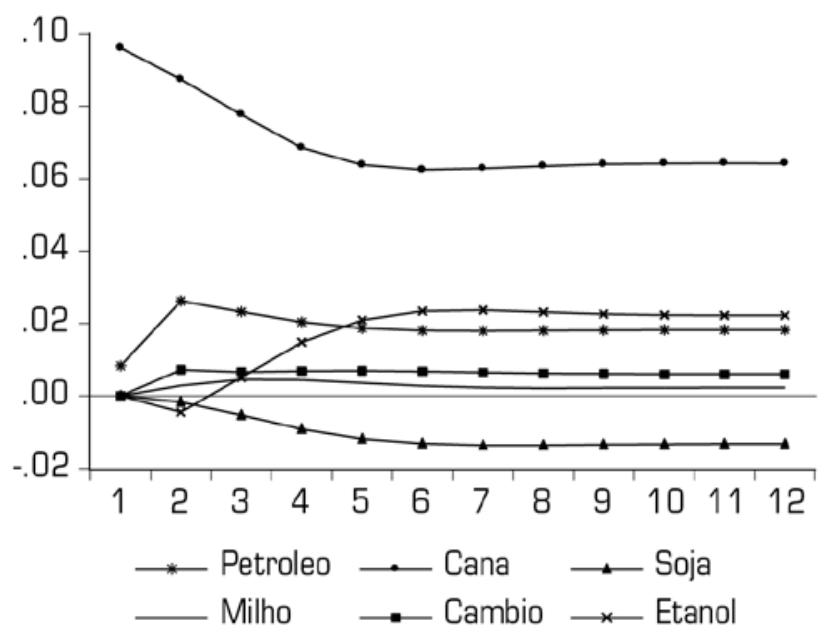

Response of Soja to Cholesky_ One S.D. Innovations

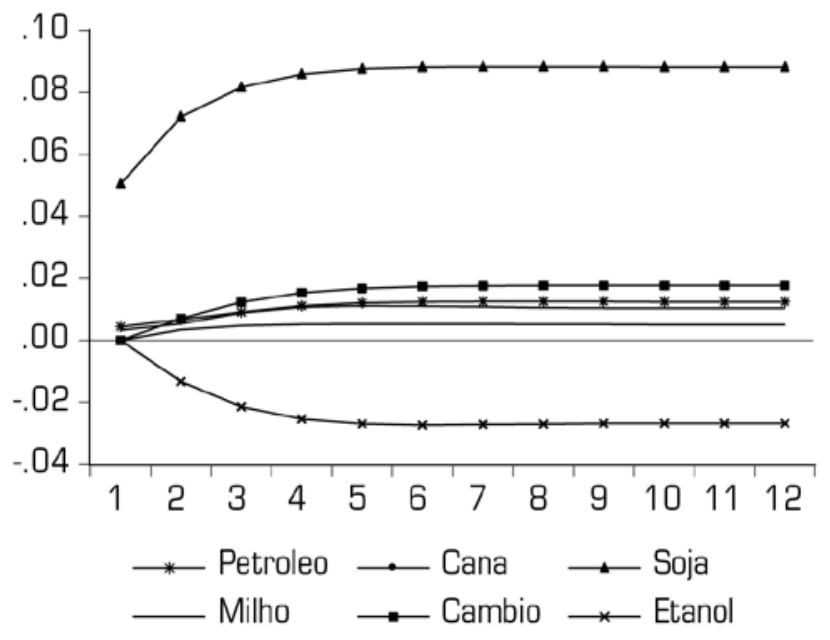

Fonte: Elaborado pelos autores.
Response of Etanol to Cholesky _ One S.D. Innovations

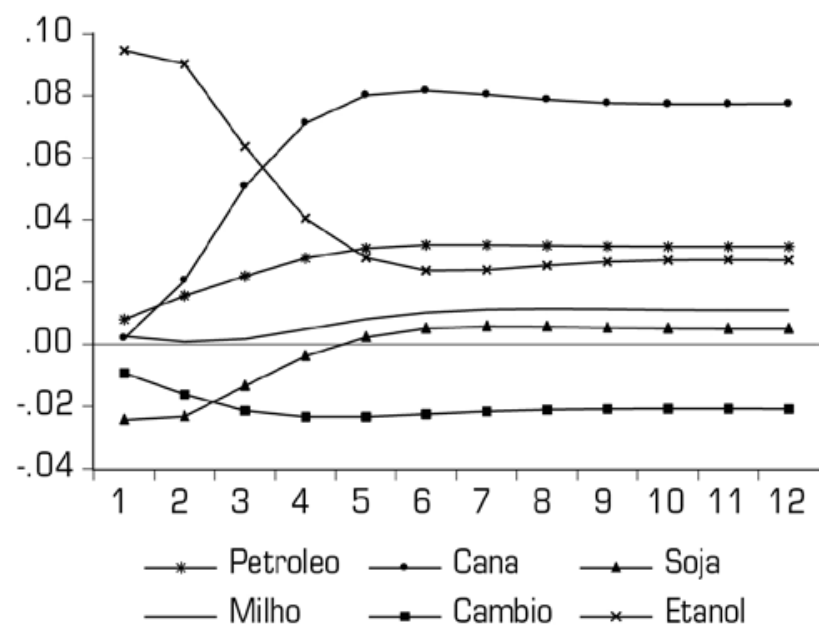

Response of Milho to Cholesky_ One S.D. Innovations

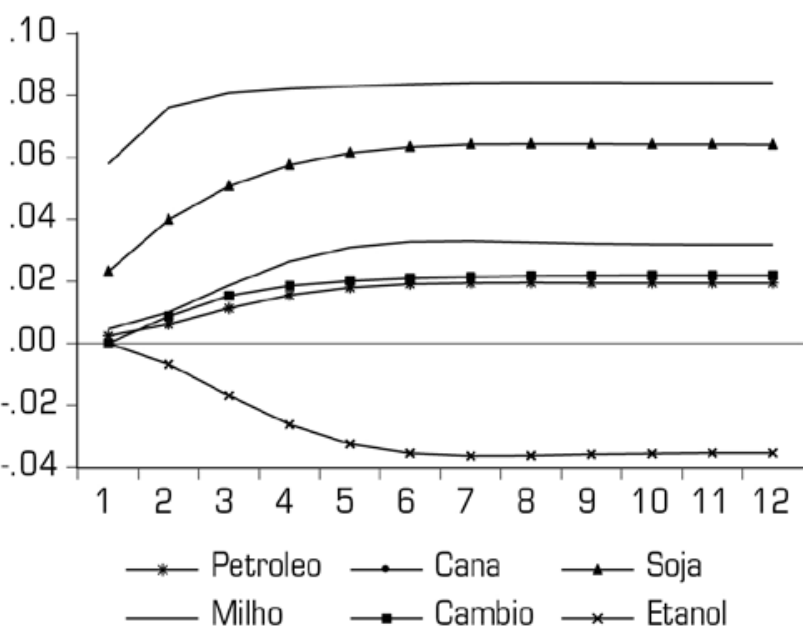


das para as commodities agrícolas. Já em relação à hipótese de integração entre as commodities agrícolas no mercado interno, essa foi parcialmente aceita. A existência de vetores cointegrantes e os resultados do teste de causalidade de Granger permitem aceitar a segunda parte da hipótese 2, de influência do mercado externo (especialmente o americano) sobre o mercado doméstico.

\section{Conclusões}

O objetivo deste trabalho foi estudar empiricamente o relacionamento entre as commodities energéticas (petróleo e etanol) e as commodities agrícolas milho e soja. Os resultados obtidos evidenciam que o preço do petróleo é uma variável importante na determinação do preço tanto do etanol como das demais commodities agrícolas. Tanto o petróleo quanto o etanol apresentaram coeficientes significativos para as commodities agrícolas. O que confirma a hipótese de dependência das commodities agrícolas em relação às fontes de energia (petróleo e etanol). Portanto, a condição de entrelaçamento entre os preços de commodities energéticas e agrícolas constatado em vários trabalhos internacionais também é valida para as condições brasileiras.

Essa dependência entre esses dois grupos de commodities é parcialmente via de mão dupla, dado que variações nos preços da cana e da soja são refletidas no preço do etanol. Também é possível confirmar a existência de interdependência entre os preços das commodities agrícolas (hipótese 2 deste estudo). $O$ aumento do preço da soja no mercado interno, puxado pelas cotações internacionais, implica menor oferta de milho e, consequentemente, elevações dos preços desse produto no mercado interno.

Como já era esperado, a taxa de câmbio tem forte relevância para os preços agrícolas no mercado brasileiro.
Também se confirmou a hipótese de "importação" de condições externas em razão da ampla inserção brasileira no mercado internacional, confirmada pela presença de vetores cointegrantes entre os preços brasileiros de milho, soja e etanol e os preços americanos de milho e etanol e pela existência de causalidade de Granger.

Por fim, a função impulso-resposta demonstrou que a resposta das variáveis aos choques não antecipados é predominantemente positiva, porém de impacto moderado, não ultrapassando variações de $10 \%$ no comportamento dos preços.

\footnotetext{
Notas

${ }^{1}$ Bolsa de Valores de Chicago.

${ }^{2} \mathrm{Na}$ existência de vetores

cointegrantes, o teste de causalidade de Granger deve ser baseado no modelo VEC estimado.
} 
AGRostat (Banco de dados). 2012. Dísponível em: https:// login.agricultura.gov.br/sso/ pages/login.jsp. Acessado em jan.2012

BAFFES, J. Oil spills on other commodities. Resources Policy, p. 126-34, 2007.

BELLINGHINI, D. F.; BELLINGHINI, P. V. M. D.; SOUZA, W.A. DA R. DE. Análise de volatilidade spillover entre commodities agrícolas e o mercado de energia: um estudo do mercado de etanol brasileiro. In conferência em gestão de risco e comercialização de commodities. Anais... São Paulo. 2011.

BLOCK, A. S.; CORONEL, D. A.; VELOSO, G. Análise da transmissão de preços no setor sucroalcooleiro brasileiro. Revista Eletrônica de Estratégia \& Negócios, v. 5, p. 120-137, 2012.

BRANDÃO, A. S. P. Commodities agrícolas e preço do petróleo: uma análise exploratória. In: CONGRESSO da Sociedade Brasileira de Economia e Sociologia Rural 48., 2012, Belo Horizonte. Anais... Belo Horizonte, SOBER, 2012.

CALDARELLI, C. E.; BACCHI, M. P. Fatores de influência no preço do milho no Brasil. Nova Economia, v. 22, n. 1, p. 141-164, 2012.
CARNEIRO, F.G. A Metodologia dos Testes de Causalidade em Economia Brasília:Universidade de Brasília (Departamento de Economia), 2012. Disponível em: www.researchgate.net/.../0046353 4836829539d000000.pdf. Acessado em: jan. 2012

CEPEA. PIB agronegócio. 2013. Disponível em: $<$ http://cepea. esalq.usp.br/pib/>. Acesso em: mar. 2013.

CHAND, R. The global food crisis: causes, severity and outlook. Review Agriculture, p. 115-122, jun. 2008.

CHRISTOFOLETTI, M. A. M.; SILVA, R. M. da. Cointegração e causalidade no mercado de soja: análises para Brasil, China e EUA. In: CONFERÊNCIA

EM GESTÃO DE RISCO E COMERCIALIZAÇÃO DE COMMODITIES, 2012, São Paulo. Anais... São Paulo, 2012.

CONAB. Perfil do setor do açúcar e do álcool no Brasil Edição para a safra 2009/2010. 2012. Disponível em: $<$ http:// www.agricultura.gov.br/arq editor/file/Desenvolvimento_ Sustentavel/Agroenergia/ estatisticas/producao/ JUNHO_2012/Publicacoes/ Perfil\%2oSucroalcoleiro\%2o -\%20safra\%202009-10.pdf $>$. Acesso em: fev. 2013.
CORONEL, D. A.; AMORIM. A. L.; de SOUSA, L. P.; de LIMA, J. E. Integração e transmissão de preços entre os mercados de trigo argentino e internacional. Pesquisa \& Debate, v. 21, n. 2, p. 279305, 2010.

CUNHA, D. A. da. A integração de preços no mercado internacional de café. Dissertação (Mestrado em Economia Aplicada) Universidade Federal de Viçosa. Viçosa 2008.

DU, X.; YU, C.; HAYES, D. Speculation and volatility spillover in the crude oil and agricultural commodity markets: a bayesian analysis. Working Paper 09-WP 491. Center for Agricultural and Rural Development, 2009. Disponível em: $<$ http://www.card.iastate. ed>. Acesso em: 30 abr. 2011.

ENDERS, W. Applied econometric time series. 2. ed. New York: John Wiley, 1995. 433 p.

FACKLER, P. L.; GOODWIN, B. K. Spatial price analysis. Chapter 17 in Handbook of Agricultural Economics (RAUSSER, G. C.) 2001, vol. 1, Part 2, pp 971-1024

FAO. Commodity market review (20092010). Roma, 2010.
FAO. Global food policy report (2011). Washington, 2012. Disponível em: <http://www.ifpri.org/ sites/default/files/publications/ gfpr2012.pdf $>$. Acesso em: mar. 2013 .

FAO. Price volatility in food and agricultural markets: Policy Responses. 2011. Disponível em: < http://www.oecd.org/tad/ agricultural-trade/48152638.pdf $>$. Acessado em Jan. 2012

GONZÁLEZ-RIVERA, G.; HELFAND, S. M. The extent, pattern and degree of market integration: a multivariate approach for the Brazilian rice market. American Journal of Agricultural Economics, v. 83, n. 3, p. 576-592, 2001.

HARRI, A., DARREN, H. Mean and variance dynamics between agricultural commodity prices and crude oil prices. In: Economics of alternative energy sources and Globalization: the road ahead meeting. Orlando, FL, 2009.

IRWIN, S. H.; GOOD, D. L. Market instability in a new era of corn, soybean, and wheat prices. Choices, Nova York, v. 24, n. 1, p. 6-11, 2009. 
LIBERA, A. A. D. Integração entre os mercados de milho e soja: uma análise através da transmissão de preços. 2009. 156 f. Dissertação (Mestrado em Agronegócios) Programa de Pós-Graduação em Agronegócio, Universidade Federal do Rio Grande do Sul, 2009.

MAPA. Brasil projeções do agronegócio brasileiro 2010/2011 a 2020/2021.

Brasília, 2011. 58p. Disponível em: $<$ http://www.agricultura.gov. br/arq_editor/file/Ministerio/ gestao/projecao/PROJECOES\%20 DO\%20AGRONEGOCIO\%20201011\%20a\%202020-21\%20-\%202_0. pdf >. Acesso em: ago. 2012.

MARGARIDO, M. A.; BUENO, C. R. F.; TUROLLA, F. A. Análise da transmissão de prelos e das volatibilidades nos mercados internacionais de petróleo e soja. In: CONGRESSO SOBER. 48., 2011, Belo Horizonte. Anais... Belo Horizonte, SOBER, 2011. p. 1-20.

MARGARIDO, Mario A.; SOUSA, Eduardo L. L.; BARBOSA, Marisa Z.; FREITAS, Silene M. Transmissão de preços no mercado internacional do grão de soja: uma aplicação da metodologia de séries temporais. In: CONGRESSO BRASILEIRO DE ECONOMIA E SOCIOLOGIA RURAL, 37., Foz do Iguaçu, 1999. Anais.... Brasília: SOBER, 1999.

MITCHELL, D. A note on rising food prices. Policy Research Working Paper No. 4682. The World Bank, Washington DC. 2008.

NERLOVE, Marc. The dynamics of supply: estimation of farmers responce to price. Baltimore: Johns Hopkins, 1958.
OLIVEIRA, A. U. de. Os agrocombustíveis e a crise dos alimentos. Jornal do Campus: Jornal da Universidade de São Paulo, São Paulo, ano 26, n. 338, 2008.

PINO, F. A.; ROCHA, M. B.

Transmissão de preços de soja no Brasil. Revista de Economia e Sociologia Rural, Brasília, v. 32, n. 4, p. 345- 61, out./dez. 1994.

ROSEGRANT, M. W. Biofuels and grain prices: impacts and policy responses. Washington. International Food Policy Research Institute, 2008.

SAGHAIAN, S. H. The impact of the oil sector on commodity prices: correlation or causation? Jurnal of Agricultural and Applied Economics, v. 42, n. 3, p. 477-485, ago. 2010.

SANTOS, R. B. N. dos; AMORIM, A. L.; CORONEL, D. A.; SANTOS, F. T. A. dos. Relações de cointegração entre preço dos biocombustíveis e alimentos: comparativo entre o etanol americano e a produção de milho no brasil. In: CONGRESSO BRASILEIRO DE ECONOMIA E SOCIOLOGIA RURAL, 50., 2010, Campo Grande. Anais.... Brasília: SOBER, 2010.

SANTOS, V. F. dos; PEREIRA, M. W. G.; VIEIRA, W. da C.

Transmissão de preços do milho entre os mercados externos e Internos. In: CONGRESSO BRASILEIRO DE ECONOMIA E SOCIOLOGIA RURAL, 45., 2007, Londrina. Anais.... Brasília: SOBER, 2007.
SERRA, T.; ZILBERMAN, D.; GIL, J. Price volatility in ethanol markets. In: ANNUAL MEETING, AGRICULTURAL AND APPLIED ECONOMICS ASSOCIATION. Milwaukee, Wisconsin,

July 26-28, 2009.

SILVA, W. V. da; SANTO, E. L.; SILVA. L. S. C. V. da. Co-integração entre os preços da soja cotados nos mercados brasileiro e norteamericano: uma análise empírica. Caderno de Pesquisas em Administração, v. 10, n. 3, p. 69-78, 2003.

TAHERIPOUR, F.; TYNER, W. E.

Ethanol policy analysis - What have we learned so far? Choices, Nova York, p. 6-11, 2008.

TYBUSCH, T. M. As estratégias de comercialização no mercado de soja: o caso da CotrijuíRS. 2003. 108 f. Dissertação (Mestrado em Agronegócios) Programa de Pós-Graduação em Agronegócios, Universidade Federal do Rio Grande do Sul, Porto Alegre, 2003.

TYNER, W.E. The integration of energy and agricultural markets. Agricultural Economics 41, 193. 2009.

VON BRAUN, J.; AHMAD, A.; OKYERE, K. A.; FAN, S.; GULATI, A.; HODDINOTT, J.; et al. High food prices: The what, who, and how of proposed policy actions. Washington. International Food Policy Research Institute, 2008.

ZHANG, Z.; LOHR, L.;

ESCALANTE, C.; WETZSTEIN,

M. Ethanol, corn, and soybean price relations in a volatile vehicle-fuels market. Energies, v. 2, p. 320-339, 2009.

\author{
E-mail de contato dos autores: \\ dienicebini@gmail.com \\ caneverm@gmail.com \\ aadenardin@gmail.com \\ Artigo recebido em abril de 2013 e \\ aprovado em março de 2014.
}


